\title{
Editorial
}

\section{Plasmonic Nanomaterials for Optical Sensor and Energy Storage and Transfer}

\author{
Xuanhua Li, ${ }^{1}$ Baofu Ding, ${ }^{2}$ Xingang Ren, ${ }^{3}$ and Yongxing Zhang ${ }^{4}$ \\ ${ }^{1}$ School of Materials Science and Engineering, Northwestern Polytechnical University, Xian, China \\ ${ }^{2}$ Department of Electrical and Electronic Engineering, Edith Cowan University, Joondalup, WA, Australia \\ ${ }^{3}$ Department of Electrical and Electronic Engineering, University of Hong Kong, Pokfulam, Hong Kong \\ ${ }^{4}$ School of Materials Science and Engineering, Huaibei Normal University, Huaibei, China
}

Correspondence should be addressed to Xuanhua Li; lixh32@nwpu.edu.cn

Received 30 May 2017; Accepted 30 May 2017; Published 22 June 2017

Copyright (C) 2017 Xuanhua Li et al. This is an open access article distributed under the Creative Commons Attribution License, which permits unrestricted use, distribution, and reproduction in any medium, provided the original work is properly cited.

Nanomaterials including noble metal nanomaterials and some metal oxide nanomaterials exhibit very strong lightmatter interactions under resonant excitation. Very large absorption and scattering at the localized wavelengths can been achieved. Because of their attractive optical properties, optical NPs and nanostructures have been commonly used in various fields from nanophotonics, analytical chemistry, biotechnology, and information storage to energy applications including photovoltaics and photocatalysis $[1,2]$.

Here, five original research articles seek to address the new preparation of optical nanomaterials, interesting design of optical sensor, and energy storage. Moreover, the new physical phenomena and mechanisms in these fields are discussed.

Dr. S. R. Tahhan and coworkers reported the fabrication of fiber Bragg grating coating with $\mathrm{TiO}_{2}$ nanostructured metal oxide for refractive index sensor. Higher shifts and narrower peaks in the Bragg wavelength were obtained after coating the fiber with few hundreds nanometers thick $\mathrm{TiO}_{2}$ coating of $20 \mathrm{~nm}-50 \mathrm{~nm}$ hole diameters. The sensitivity of the sensor with the $\mathrm{TiO}_{2}$ coating is higher than that without coating due to the nanostructure's large surface area.

The mode structures of multiphoton induced ultraviolet laser in a $\mathrm{ZnO}$ microrod have been investigated by Dr. G. $\mathrm{Zhu}$. Hexagonal wurtzite structural $\mathrm{ZnO}$ microrods were fabricated by vapor-phase transport method. Under the excitation of a pulse laser with $1200 \mathrm{~nm}$ wavelength, the multiphoton induced ultraviolet (UV) laser was observed in a microrod. The dependence of the laser mode structures on pump intensity was investigated. The result indicates that the laser belongs to whispering gallery mode (WGM) at low pump intensity and Fabry-Perot (FP) mode at high pump intensity.

Another work around controllable growth of the $\mathrm{ZnO}$ nanorod arrays has been reported by Dr. Q. Liu and coworkers. High quality $\mathrm{ZnO}$ nanorod arrays are formed using the $\mathrm{ZnO}$ nanoflakes on the $\mathrm{Al}$ substrates as seed layer. They found that a reversible wettability transition can be easily achieved via alternation of UV irradiation and dark storage. The physical adsorption of the water molecules on the surface of $\mathrm{ZnO}$ nanorod arrays is considered to be responsible for this transition, which is confirmed by $\mathrm{X}$-ray photoelectron spectroscopy.

L. Cai and Dr. C. Feng have discussed the effect of vacancy defects on the electronic structure and optical properties of $\mathrm{GaN}$ using the generalized gradient approximation method within the density functional theory. The results show that the band gap increases in $\mathrm{GaN}$ with vacancy defects. Crystal parameters decrease in $\mathrm{GaN}$ with nitrogen vacancy (GaN:VN) and increase in GaN with gallium vacancy (GaN:VGa). The Ga vacancy introduces defect levels at the top of the valence band, and the defect levels are contributed by $\mathrm{N} 2 \mathrm{p}$ electron states.

In addition, synthesis of $x \mathrm{LiMnPO} 4 \cdot y \mathrm{Li} 3 \mathrm{~V} 2(\mathrm{PO} 4) 3 / \mathrm{C}$ nanocomposites for lithium-ion batteries using tributyl phosphate as phosphor source has been reported by Dr. F. Wang and coworkers. They synthesized new $x$ LiMnPO4.yLi3V2 (PO4)3/C nanocomposites, which is a very important issue for high-performance lithium-ion batteries.

The topic around optical properties of nanomaterials is very important and timely. They have been widely applied 
in plasmon-enhanced spectroscopy, photocatalysis, energy storage, and solar cells. This topic is of relevance to the general research in chemistry, physics, optics, and material science and will attract great attentions of researchers in these fields who are new to or experienced in the plasmon-enhanced application in wide fields. Therefore, the potential broad audience of this issue will further promote the development of plasmon-related researches and accelerate their practical applications in various fields including surface-enhanced spectroscopy, energy storage, and energy transfer.

Xuanhua Li

Baofu Ding

Xingang Ren

Yongxing Zhang

\section{References}

[1] S. Guo, X. Li, J. Zhu, T. Tong, and B. Wei, “Au NPs@MoS submicrometer sphere- $\mathrm{ZnO}$ nanorod hybrid structures for efficient photocatalytic hydrogen evolution with excellent stability," Small, vol. 12, no. 41, pp. 5692-5701, 2016.

[2] X. Li, S. Guo, C. Kan et al., "Au Multimer@MoS2 hybrid structures for efficient photocatalytical hydrogen production via strongly plasmonic coupling effect," Nano Energy, vol. 30, pp. 549-558, 2016. 

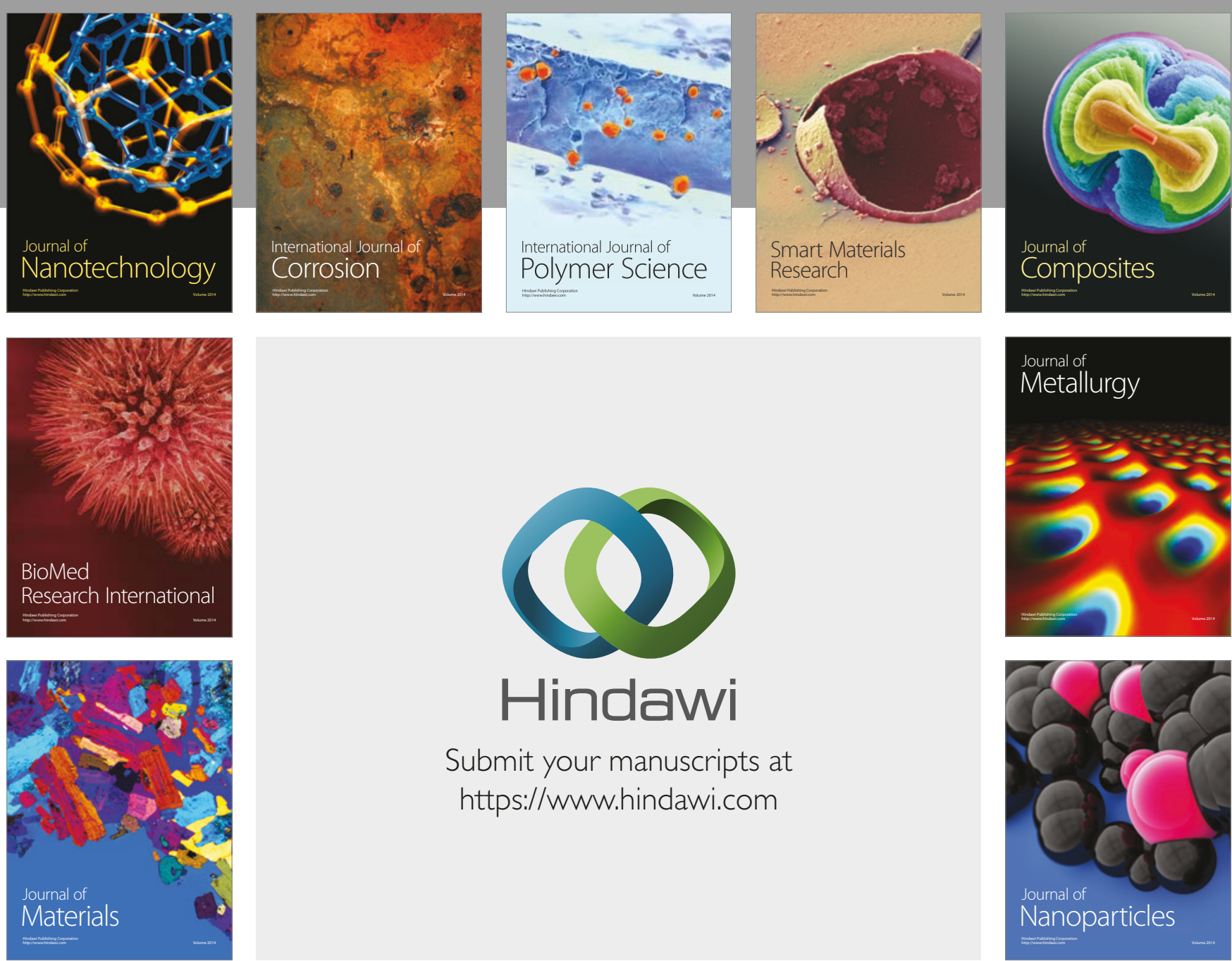

\section{Hindawi}

Submit your manuscripts at

https://www.hindawi.com
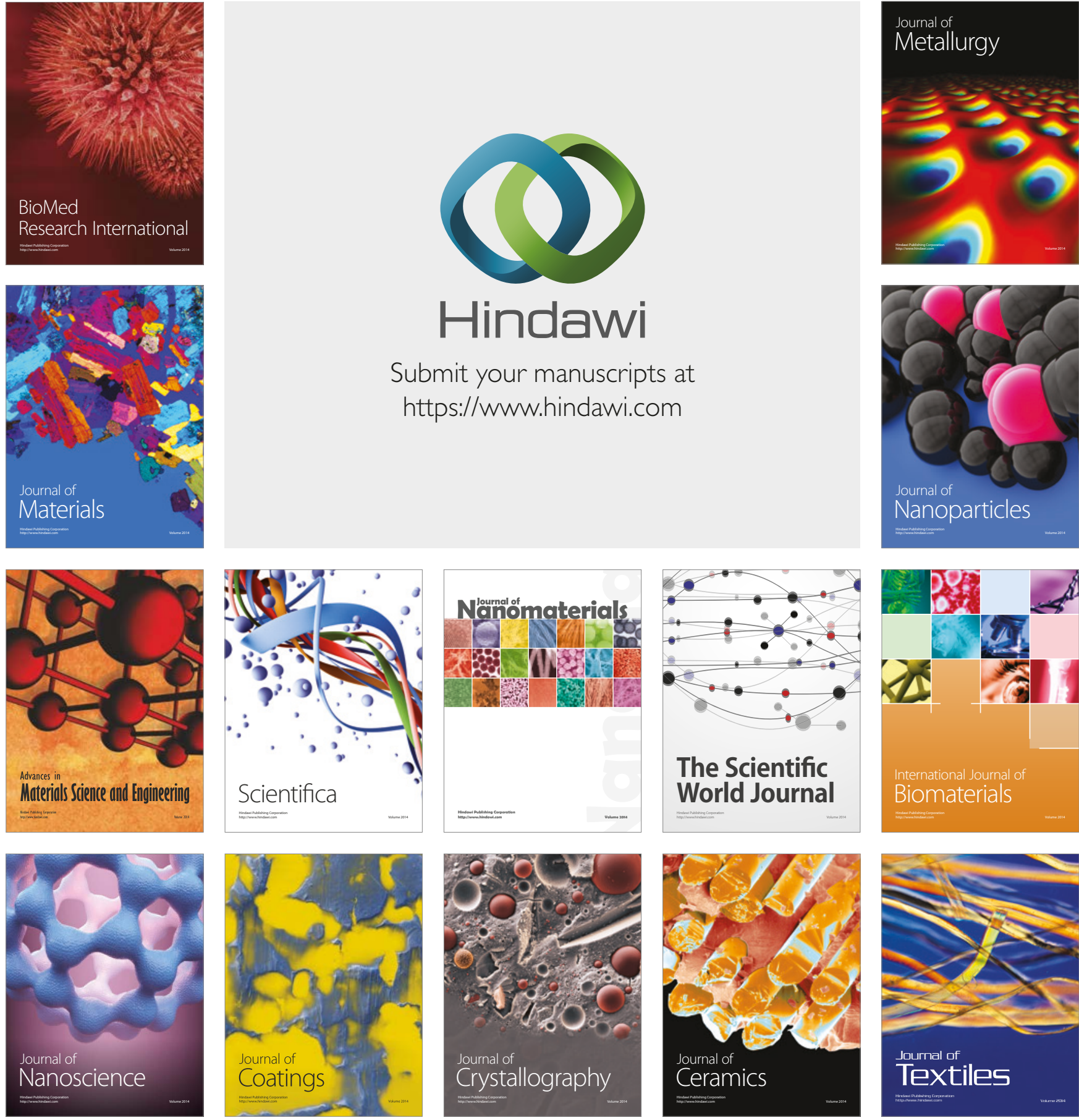

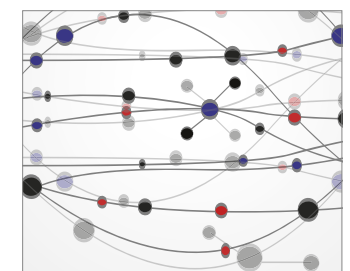

The Scientific World Journal
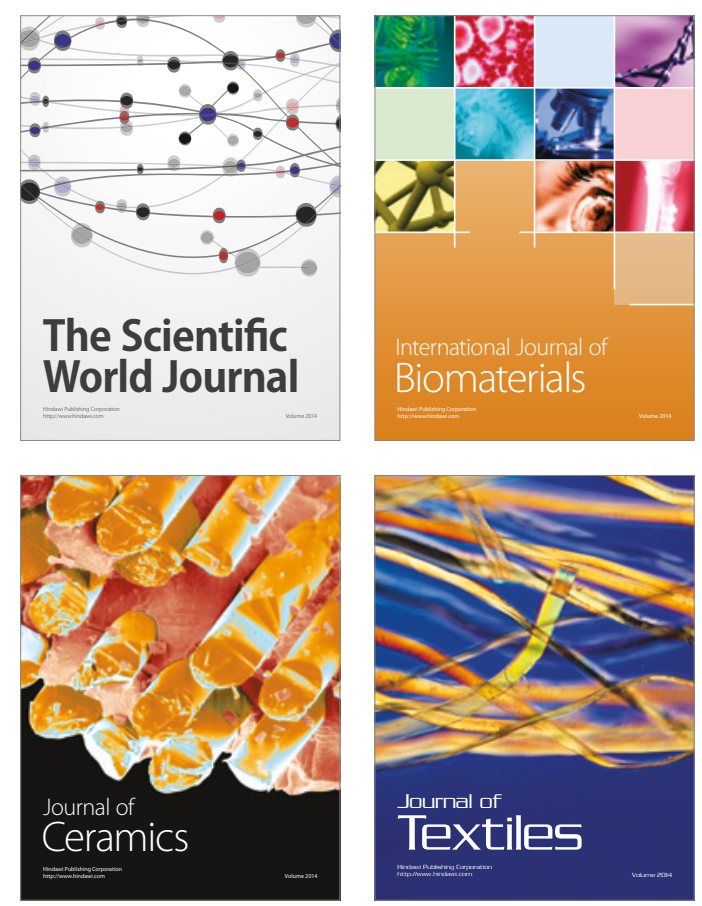